\title{
Nonlinear Super Integrable Couplings of a Super Integrable Hierarchy
}

\author{
Sixing Tao \\ School of Mathematics and Information Science, Shangqiu Normal University, Shangqiu, China \\ Email: taosixing@163.com
}

Received 1 March 2016; accepted 6 April 2016; published 13 April 2016

\begin{abstract}
Nonlinear super integrable couplings of a super integrable hierarchy based upon an enlarged matrix Lie super algebra were constructed. And its super Hamiltonian structures were established by using super trace identity. As its reduction, special cases of this nonlinear super integrable coupling were obtained.
\end{abstract}

\section{Keywords}

\section{Lie Super Algebra, Nonlinear Super Integrable Couplings, A Super Integrable Hierarchy, Super Hamiltonian Structures}

\section{Introduction}

With the development of soliton theory, super integrable systems associated with Lie super algebra have aroused growing attentions by many mathematicians and physicists. It was known that super integrable systems contained the odd variables, which would provide more prolific fields for mathematical researchers and physical ones. Several super integrable systems including super AKNS hierarchy, super KdV hierarchy, super KP hierarchy, etc., have been studied in [1]-[4]. There are some interesting results on the super integrable systems, such as Darboux transformation in [5], super Hamiltonian structures in [6] [7], binary nonlinearization [8] and reciprocal transformation [9] and so on.

The research of integrable couplings of the well known integrable hierarchy has received considerable attention [10]-[12]. A few approaches to construct linear integrable couplings of the classical soliton equation are presented by permutation, enlarging spectral problem, using matrix Lie algebra [13] constructing new loop Lie algebra and creating semi-direct sums of Lie algebra. Recently, You [14] presented a scheme for constructing the nonlinear super integrable couplings for the super integrable hierarchy. Zhang [15] once constructed an integrable hierarchy and discussed Lax representation, Darboux transformation for its constrained flows. Shi [16] constructed the super extension of this hierarchy.

In this paper, we hope to construct nonlinear super integrable couplings of this super integrable hierarchy which was constructed in [16] through enlarging matrix Lie super algebra. We take the Lie algebra $B(0,1)$ as an example to illustrate the approach for extending Lie super algebras. Based on the enlarged Lie super algebra $g l(6,2)$, we work out nonlinear super integrable Hamiltonian couplings of this super integrable hierarchy. Finally, we will reduce the nonlinear super integrable couplings to some special cases. 


\section{Enlargement of Lie Super Algebra $B(0,1)$}

Consider the Lie super algebra $B(0,1)$. Its basis is

$$
E_{1}=\left(\begin{array}{lll}
0 & 1 & 0 \\
1 & 0 & 0 \\
0 & 0 & 0
\end{array}\right), E_{2}=\left(\begin{array}{ccc}
0 & 1 & 0 \\
-1 & 0 & 0 \\
0 & 0 & 0
\end{array}\right), E_{3}=\left(\begin{array}{ccc}
1 & 0 & 0 \\
0 & -1 & 0 \\
0 & 0 & 0
\end{array}\right), E_{4}=\left(\begin{array}{ccc}
0 & 0 & 1 \\
0 & 0 & 0 \\
0 & -1 & 0
\end{array}\right), E_{5}=\left(\begin{array}{ccc}
0 & 0 & 0 \\
0 & 0 & 1 \\
1 & 0 & 0
\end{array}\right) .
$$

where $E_{1}, E_{2}, E_{3}$ are even element and $E_{4}, E_{5}$ are odd elements. Their non-zero (anti) commutation relations are

$$
\begin{aligned}
& {\left[E_{1}, E_{2}\right]=-2 E_{3},\left[E_{1}, E_{3}\right]=-2 E_{2},\left[E_{1}, E_{4}\right]=E_{5},\left[E_{1}, E_{5}\right]=E_{4},\left[E_{2}, E_{3}\right]=-2 E_{1},\left[E_{2}, E_{4}\right]=-E_{5},\left[E_{2}, E_{5}\right]=E_{4},} \\
& {\left[E_{3}, E_{4}\right]=E_{4},\left[E_{3}, E_{5}\right]=-E_{5},\left[E_{4}, E_{4}\right]=-\left(E_{1}+E_{2}\right),\left[E_{4}, E_{5}\right]=\left[E_{5}, E_{4}\right]=E_{3},\left[E_{5}, E_{5}\right]=E_{1}-E_{2} .}
\end{aligned}
$$

Let us enlarge the Lie super algebra $B(0,1)$ to the Lie super algebra $g l(6,2)$ with a basis

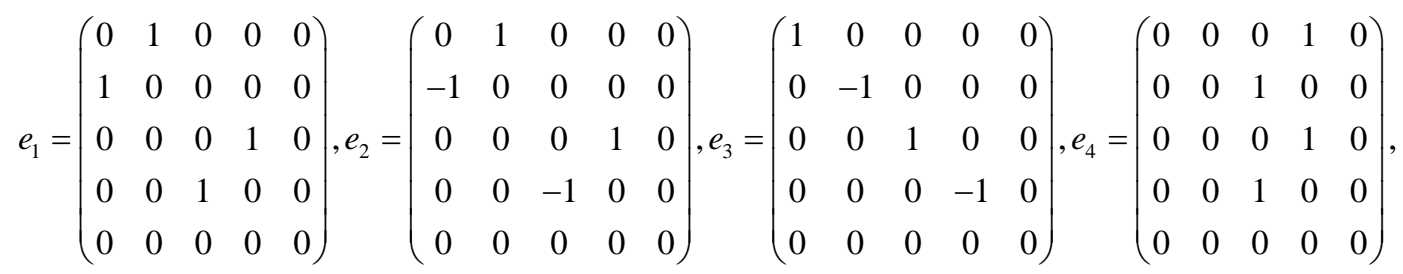

$$
\begin{aligned}
& e_{5}=\left(\begin{array}{ccccc}
0 & 0 & 0 & 1 & 0 \\
0 & 0 & -1 & 0 & 0 \\
0 & 0 & 0 & 1 & 0 \\
0 & 0 & -1 & 0 & 0 \\
0 & 0 & 0 & 0 & 0
\end{array}\right), e_{6}=\left(\begin{array}{ccccc}
0 & 0 & 1 & 0 & 0 \\
0 & 0 & 0 & -1 & 0 \\
0 & 0 & 1 & 0 & 0 \\
0 & 0 & 0 & -1 & 0 \\
0 & 0 & 0 & 0 & 0
\end{array}\right), e_{7}=\left(\begin{array}{ccccc}
0 & 0 & 0 & 0 & 1 \\
0 & 0 & 0 & 0 & 0 \\
0 & 0 & 0 & 0 & 0 \\
0 & 0 & 0 & 0 & 0 \\
0 & -1 & 0 & 1 & 0
\end{array}\right), e_{8}=\left(\begin{array}{ccccc}
0 & 0 & 0 & 0 & 0 \\
0 & 0 & 0 & 0 & 1 \\
0 & 0 & 0 & 0 & 0 \\
0 & 0 & 0 & 0 & 0 \\
1 & 0 & -1 & 0 & 0
\end{array}\right) .
\end{aligned}
$$

where $e_{1}, e_{2}, e_{3}, e_{4}, e_{5}, e_{6}$ are even, and $e_{7}, e_{8}$ are odd.

The generator of Lie super algebra $g l(6,2), e_{i}(1 \leq i \leq 8)$ satisfy the following (anti) commutation relations:

$$
\begin{aligned}
& {\left[e_{1}, e_{2}\right]=-2 e_{3},\left[e_{1}, e_{3}\right]=-2 e_{2},\left[e_{1}, e_{5}\right]=-2 e_{6},\left[e_{1}, e_{6}\right]=-2 e_{5},\left[e_{1}, e_{7}\right]=e_{8},\left[e_{1}, e_{8}\right]=e_{7},\left[e_{2}, e_{3}\right]=-2 e_{1},\left[e_{2}, e_{4}\right]=2 e_{6},} \\
& {\left[e_{2}, e_{6}\right]=-2 e_{4},\left[e_{2}, e_{7}\right]=-e_{8},\left[e_{2}, e_{8}\right]=e_{7},\left[e_{3}, e_{4}\right]=2 e_{5},\left[e_{3}, e_{5}\right]=2 e_{4},\left[e_{3}, e_{7}\right]=e_{7},\left[e_{3}, e_{8}\right]=-e_{8},\left[e_{4}, e_{5}\right]=-2 e_{6},} \\
& {\left[e_{4}, e_{6}\right]=-2 e_{5},\left[e_{5}, e_{6}\right]=-2 e_{4},\left[e_{7}, e_{7}\right]=-e_{1}-e_{2}+e_{4}+e_{5},\left[e_{7}, e_{8}\right]=\left[e_{8}, e_{7}\right]=e_{3}-e_{6},\left[e_{8}, e_{8}\right]=e_{1}-e_{2}-e_{4}+e_{5},} \\
& {\left[e_{1}, e_{4}\right]=\left[e_{2}, e_{5}\right]=\left[e_{3}, e_{6}\right]=\left[e_{4}, e_{7}\right]=\left[e_{4}, e_{8}\right]=\left[e_{5}, e_{7}\right]=\left[e_{5}, e_{8}\right]=\left[e_{6}, e_{7}\right]=\left[e_{6}, e_{8}\right]=0 .}
\end{aligned}
$$

Define a loop super algebra corresponding to the Lie super algebra $g l(6,2)$, denote by

$$
\widetilde{g l}(6,2)=g l(6,2) \otimes \mathbb{C}\left[\lambda, \lambda^{-1}\right]=\left\{e_{i} \lambda^{m}, e_{i} \in g l(6,2), i=1,2, \cdots, 8 ; m=0, \pm 1, \pm 2, \cdots .\right.
$$

The corresponding (anti)commutative relations are given as

$$
\left[e_{i} \lambda^{m}, e_{j} \lambda^{n}\right]=\left[e_{i}, e_{j}\right] \lambda^{m+n}, \forall e_{i}, e_{j} \in g l(6,2) .
$$

\section{Nonlinear Super Integrable Couplings of a Super Integrable Hierarchy}

If Let us start from an enlarged spectral problem associated with $g l(6,2)$,

$$
\begin{aligned}
& \phi_{x}=U(u, \lambda) \phi, U=e_{1}(1)+q e_{2}(0)+r e_{3}(0)+u_{1} e_{5}(0)+u_{2} e_{6}(0)+\alpha e_{7}(0)+\beta e_{8}(0) \\
& =\left(\begin{array}{ccccc}
r & \lambda+q & u_{2} & u_{1} & \alpha \\
\lambda-q & -r & -u_{1} & -u_{2} & \beta \\
0 & 0 & r+u_{2} & \lambda+q+u_{1} & 0 \\
0 & 0 & \lambda-q-u_{1} & -r-u_{2} & 0 \\
\beta & -\alpha & -\beta & \alpha & 0
\end{array}\right) .
\end{aligned}
$$


where $q, r, u_{1}, u_{2}$ are even potentials, but $\alpha, \beta$ are odd ones.

In order to obtain super integrable couplings of super integrable hierarchy, we solve the adjoint representation of (7),

$$
V_{x}=[U, V],
$$

with

$$
\begin{aligned}
& V=A e_{1}(0)+B e_{2}(0)+C e_{3}(0)+E e_{4}(0)+F e_{5}(0)+G e_{6}(0)+\rho e_{7}(0)+\delta e_{8}(0) \\
& =\left(\begin{array}{ccccc}
C & A+B & G & E+F & \rho \\
A-B & -C & E-F & -G & \delta \\
0 & 0 & C+G & A+B+E+F & 0 \\
0 & 0 & A-B+E-F & -A-E & 0 \\
\delta & -\rho & -\delta & \rho & 0
\end{array}\right)
\end{aligned}
$$

where $A, B, C, E, F$ and $G$ are commuting fields, and $\rho, \delta$ are anti-commuting fields.

Substituting

$$
\begin{aligned}
& A=\sum_{m \geq 0} A_{m} \lambda^{-m}, B=\sum_{m \geq 0} B_{m} \lambda^{-m}, C=\sum_{m \geq 0} C_{m} \lambda^{-m}, E=\sum_{m \geq 0} E_{m} \lambda^{-m}, \\
& F=\sum_{m \geq 0} F_{m} \lambda^{-m}, G=\sum_{m \geq 0} G_{m} \lambda^{-m}, \rho=\sum_{m \geq 0} \rho_{m} \lambda^{-m}, \delta=\sum_{m \geq 0} \delta_{m} \lambda^{-m} .
\end{aligned}
$$

into previous equation gives the following recursive formulas

$$
\left\{\begin{array}{l}
A_{m, x}=2 r B_{m}-2 q C_{m}-\alpha \rho_{m}+\beta \delta_{m}, \\
B_{m, x}=2 r A_{m}-2 C_{m+1}-\alpha \rho_{m}-\beta \delta_{m}, \\
C_{m, x}=2 q A_{m}-2 B_{m+1}+\beta \rho_{m}+\alpha \delta_{m}, \\
E_{m, x}=2 u_{2} B_{m}-2 u_{1} C_{m}+2 r F_{m}+2 u_{2} F_{m}-2 q G_{m}-2 u_{1} G_{m}+\alpha \rho_{m}-\beta \delta_{m}, \\
F_{m, x}=2 u_{2} A_{m}+2 r E_{m}+2 u_{2} E_{m}-2 G_{m+1}+\alpha \rho_{m}+\beta \delta_{m}, \\
G_{m, x}=2 u_{1} A_{m}+2 q E_{m}+2 u_{1} E_{m}-2 F_{m+1}-\beta \rho_{m}-\alpha \delta_{m}, \\
\rho_{m, x}=-\beta A_{m}-\beta B_{m}-\alpha C_{m}+r \rho_{m}+q \delta_{m}+\delta_{m+1}, \\
\delta_{m, x}=-\alpha A_{m}+\alpha B_{m}+\beta C_{m}-q \rho_{m}+\rho_{m+1}-r \delta_{m} .
\end{array}\right.
$$

From previous equations, we can successively deduce

$$
\begin{aligned}
& A_{0}=1, B_{0}=C_{0}=F_{0}=G_{0}=\rho_{0}=\delta_{0}=0, E_{0}=\varepsilon=\text { const., } A_{1}=0, B_{1}=q, C_{1}=r, E_{1}=0, F_{1}=\varepsilon q+u_{1}+\varepsilon u_{1}, \\
& G_{1}=\varepsilon r+u_{2}+\varepsilon u_{2}, \rho_{1}=\alpha, \delta_{1}=\beta, A_{2}=\frac{1}{2} q^{2}-\frac{1}{2} r^{2}-\alpha \beta, B_{2}=-\frac{1}{2} r_{x}, C_{2}=-\frac{1}{2} q_{x}, E_{2}=\frac{1}{2} \varepsilon q^{2}-\frac{1}{2} \varepsilon r^{2} \\
& +(1+\varepsilon)\left(q u_{1}-r u_{2}+\frac{1}{2} u_{1}^{2}-\frac{1}{2} u_{2}{ }^{2}\right)+\alpha \beta, F_{2}=-\frac{1}{2} \varepsilon r_{x}-\frac{1}{2} u_{2 x}-\frac{1}{2} \varepsilon u_{2 x}, G_{2}=-\frac{1}{2} \varepsilon q_{x}-\frac{1}{2} u_{1 x}-\frac{1}{2} \varepsilon u_{1 x}, \\
& \rho_{2}=\beta_{x}, \delta_{2}=\alpha_{x}, A_{3}=\frac{1}{2} q_{x} r-\frac{1}{2} q r_{x}-\alpha \alpha_{x}+\beta \beta_{x}, B_{3}=\frac{1}{4} q_{x x}+\frac{1}{2} q^{3}-\frac{1}{2} q r^{2}-q \alpha \beta+\frac{1}{2} \alpha \alpha_{x}+\frac{1}{2} \beta \beta_{x}, \\
& C_{3}=\frac{1}{4} r_{x x}+\frac{1}{2} q^{2} r-\frac{1}{2} r^{3}-r \alpha \beta-\frac{1}{2} \alpha \beta_{x}+\frac{1}{2} \alpha_{x} \beta, E_{3}=\frac{1}{2} \varepsilon q_{x} r-\frac{1}{2} \varepsilon q r_{x}+(1+\varepsilon)\left(\frac{1}{2} r u_{1}+\frac{1}{2} u_{1 x} u_{2}\right. \\
& \left.-\frac{1}{2} u_{1} u_{2 x}-\frac{1}{2} r_{x} u_{1}+\frac{1}{2} q_{x} u_{2}-\frac{1}{2} q u_{2 x}\right)+\alpha \alpha_{x}-\beta \beta_{x}, F_{3}=\frac{1}{4} \varepsilon q_{x x}+\frac{1}{2} \varepsilon q^{3}-\frac{1}{2} \varepsilon q r^{2}+(1+\varepsilon)\left(\frac{1}{4} u_{1 x x}\right. \\
& \left.-q r u_{2}-r u_{1} u_{2}-\frac{1}{2} r^{2} u_{1}+\frac{3}{2} q u_{1}^{2}+\frac{3}{2} q^{2} u_{1}-\frac{1}{2} u_{1} u_{2}^{2}-\frac{1}{2} q u_{2}^{2}+\frac{1}{2} u_{1}^{3}\right)+q \alpha \beta-\frac{1}{2} \alpha \alpha_{x}-\frac{1}{2} \beta \beta_{x}, \\
& G_{3}=\frac{1}{4} \varepsilon r_{x x}+\frac{1}{2} \varepsilon q^{2} r-\frac{1}{2} \varepsilon r^{3}+(1+\varepsilon)\left(\frac{1}{4} u_{2 x x}+q r u_{1}+q u_{1} u_{2}-\frac{3}{2} r u_{2}^{2}-\frac{3}{2} r^{2} u_{2}+\frac{1}{2} q^{2} u_{2}+\frac{1}{2} u_{1}^{2} u_{2}\right. \\
& \left.+\frac{1}{2} r u_{1}^{2}-\frac{1}{2} u_{2}^{3}\right)+r \alpha \beta+\frac{1}{2} \alpha \beta_{x}-\frac{1}{2} \alpha_{x} \beta, \rho_{3}=\alpha_{x x}+\frac{1}{2} r_{x} \alpha+\frac{1}{2} q_{x} \beta+\frac{1}{2} q^{2} \alpha-\frac{1}{2} r^{2} \alpha+r \alpha_{x}+q \beta_{x}, \\
& \delta_{3}=\beta_{x x}-\frac{1}{2} q_{x} \alpha-\frac{1}{2} r_{x} \beta+\frac{1}{2} q^{2} \beta-\frac{1}{2} r^{2} \beta-q \alpha_{x}-r \beta_{x} .
\end{aligned}
$$


Equations (11) can be written as

$$
\left(\begin{array}{c}
-2 B_{m+1}-F_{m+1} \\
2 C_{m+1}+G_{m+1} \\
-B_{m+1}-F_{m+1} \\
C_{m+1}+G_{m+1} \\
\delta_{m+1} \\
-\rho_{m+1}
\end{array}\right)=L\left(\begin{array}{c}
-2 B_{m}-F_{m} \\
2 C_{m}+G_{m} \\
-B_{m}-F_{m} \\
C_{m}+G_{m} \\
\delta_{m} \\
-\rho_{m}
\end{array}\right)
$$

where

$$
L=\left(\begin{array}{cccccc}
2 q \partial^{-1} r & 2 q \partial^{-1} q+\frac{1}{2} \partial & 2 u_{1} \partial^{-1} r+2 q \partial^{-1} u_{2}+2 u_{1} \partial^{-1} u_{2} & 2 u_{1} \partial^{-1} q+2 q \partial^{-1} u_{1}+2 u_{1} \partial^{-1} u_{1} & -\frac{1}{2} \partial-q \partial^{-1} \beta & \frac{1}{2} \beta-q \partial^{-1} \alpha \\
\frac{1}{2} \partial-2 r \partial^{-1} r & -2 r \partial^{-1} q & -2 r \partial^{-1} u_{2}-2 u_{2} \partial^{-1} r-2 u_{2} \partial^{-1} u_{2} & -2 r \partial^{-1} u_{1}-2 u_{2} \partial^{-1} q-2 u_{2} \partial^{-1} u_{1} & r \partial^{-1} \beta-\frac{1}{2} \beta & \frac{1}{2} \alpha+r \partial^{-1} \alpha \\
0 & 0 & 2 q \partial^{-1} r+2 q \partial^{-1} u_{2}+2 u_{1} \partial^{-1} r+2 u_{1} \partial^{-1} u_{2} & \frac{1}{2} \partial+2 q \partial^{-1} u_{1}+2 q \partial^{-1} q+2 u_{1} \partial^{-1} q+2 u_{1} \partial^{-1} u_{1} & 0 & 0 \\
0 & 0 & \frac{1}{2} \partial-2 r \partial^{-1} r-2 r \partial^{-1} u_{2}-2 u_{2} \partial^{-1} r-2 u_{2} \partial^{-1} u_{2} & -2 r \partial^{-1} q-2 r \partial^{-1} u_{1}-2 u_{2} \partial^{-1} q-2 u_{2} \partial^{-1} u_{1} & 0 & 0 \\
-\beta-2 \beta \partial^{-1} r & \alpha-2 \beta \partial^{-1} q & \beta+2 \beta \partial^{-1} r & -\alpha+2 \beta \partial^{-1} q & \beta \partial^{-1} \beta-q & r-\partial+\beta \partial^{-1} \alpha \\
-\alpha+2 \alpha \partial^{-1} r & \beta+2 \alpha \partial^{-1} q & \alpha-2 \alpha \partial^{-1} r & -\beta-2 \alpha \partial^{-1} q & -r-\partial-\alpha \partial^{-1} \beta & q-\alpha \partial^{-1} \alpha
\end{array}\right) .
$$

Then, let us consider the spectral problem (7) with the following auxiliary problem

$$
\phi_{t_{n}}=V^{(n)} \phi=\sum_{j=0}^{n}\left(\begin{array}{ccccc}
C_{j} & A_{j}+B_{j} & G_{j} & E_{j}+F_{j} & \rho_{j} \\
A_{j}-B_{j} & -C_{j} & E_{j}+F_{j} & -G_{j} & \delta_{j} \\
0 & 0 & C_{j}+G_{j} & A_{j}+B_{j}+E_{j}+F_{j} & 0 \\
0 & 0 & A_{j}-B_{j}+E_{j}-F_{j} & -C_{j}-G_{j} & 0 \\
\delta_{j} & -\rho_{j} & -\delta_{j} & \rho_{j} & 0
\end{array}\right) \lambda^{n-j} \phi,
$$

From the compatible condition $\phi_{x, t_{n}}=\phi_{t_{n}, x}$, according to (7) and (14), we get the zero curvature equation

$$
U_{t_{n}}-V_{x}^{(n)}+\left[U, V^{(n)}\right]=0 .
$$

which gives a nonlinear Lax super integrable hierarchy

$$
u_{t_{n}}=\left(\begin{array}{c}
q \\
r \\
u_{1} \\
u_{2} \\
\alpha \\
\beta
\end{array}\right)_{t_{n}}=\left(\begin{array}{c}
-2 C_{n+1} \\
-2 B_{n+1} \\
-2 G_{n+1} \\
-2 F_{n+1} \\
\delta_{n+1} \\
\rho_{n+1}
\end{array}\right)
$$

The super integrable hierarchy (16) is a nonlinear super integrable couplings for the integrable hierarchy in [16]

$$
\tilde{u}_{t_{n}}=\left(\begin{array}{c}
q \\
r \\
\alpha \\
\beta
\end{array}\right)_{t_{n}}=\left(\begin{array}{c}
-2 C_{n+1} \\
-2 B_{n+1} \\
\delta_{n+1} \\
\rho_{n+1}
\end{array}\right) .
$$

\section{Super Hamiltonian Structure}

A direct calculation reads

$$
\begin{aligned}
& \operatorname{Str}\left(U_{\lambda}, V\right)=4 A+2 E, \operatorname{Str}\left(U_{q}, V\right)=-4 B-2 F, \operatorname{Str}\left(U_{r}, V\right)=4 C+2 G, \\
& \operatorname{Str}\left(U_{u_{1}}, V\right)=-2 B-2 F, \operatorname{Str}\left(U_{u_{2}}, V\right)=2 C+2 G, \operatorname{Str}\left(U_{\alpha}, V\right)=2 \delta, \operatorname{Str}\left(U_{\beta}, V\right)=-2 \rho .
\end{aligned}
$$


Substituting above results into the super trace identity [7]

$$
\frac{\delta}{\delta u} \int \operatorname{Str}\left(\frac{\delta U}{\delta \lambda} V\right) \mathrm{d} x=\lambda^{-\gamma} \frac{\partial}{\partial \lambda} \lambda^{\gamma} \operatorname{Str}\left(\frac{\delta U}{\delta u} V\right),
$$

and comparing the coefficients of $\lambda^{-n-1}$ on both side of (19)

$$
\frac{\delta}{\delta u} \int\left(4 A_{n+1}+2 E_{n+1}\right) \mathrm{d} x=\lambda^{-\gamma} \frac{\partial}{\partial \lambda} \lambda^{\gamma}\left(\begin{array}{c}
-4 B_{n}-2 F_{n} \\
4 C_{n}+2 G_{n} \\
-2 B_{n}-2 F_{n} \\
2 C_{n}+2 G_{n} \\
2 \delta_{n} \\
-2 \rho_{n}
\end{array}\right), n \geq 0 .
$$

From the initial values in (11), we obtain $\gamma=0$. Thus we have

$$
\frac{\delta H_{n}}{\delta u}=\left(\begin{array}{c}
-2 B_{n}-F_{n} \\
2 C_{n}+G_{n} \\
-B_{n}-F_{n} \\
C_{n}+G_{n} \\
\delta_{n} \\
-\rho_{n}
\end{array}\right), H_{n}=-\int \frac{2 A_{n+1}+E_{n+1}}{n+1} \mathrm{~d} x, n \geq 0 .
$$

It then follows that the nonlinear super integrable couplings (16) possess the following super Hamiltonian form

where

$$
u_{t_{n}}=K_{n}(u)=J \frac{\delta H_{n}}{\delta u}
$$

$$
J=\left(\begin{array}{cccccc}
0 & -2 & 0 & 2 & 0 & 0 \\
2 & 0 & -2 & 0 & 0 & 0 \\
0 & 2 & 0 & -4 & 0 & 0 \\
-2 & 0 & 4 & 0 & 0 & 0 \\
0 & 0 & 0 & 0 & 1 & 0 \\
0 & 0 & 0 & 0 & 0 & -1
\end{array}\right) .
$$

is a super Hamiltonian operator and $H_{n}(n \geq 0)$ are Hamiltonian functions.

\section{Reductions}

Taking $\alpha=\beta=0$, (16) reduces to a nonlinear integrable couplings of the integrable hierarchy in [15].

When $n=2$ in (16), we obtain the nonlinear super integrable couplings of the second order super integrable equations

$$
\left\{\begin{aligned}
q_{t_{2}}= & -\frac{1}{2} r_{x x}-q^{2} r+r^{3}+2 r \alpha \beta+\alpha \beta_{x}-\alpha_{x} \beta, \\
r_{t_{2}}= & -\frac{1}{2} q_{x x}-q^{3}+q r^{2}+2 q \alpha \beta-\alpha \alpha_{x}-\beta \beta_{x}, \\
u_{1, t_{2}}= & -\frac{1}{2} \varepsilon r_{x x}-\varepsilon q^{2} r+\varepsilon r^{3}+(\varepsilon+1)\left(-\frac{1}{2} u_{2 x x}-2 q r u_{1}-2 q u_{1} u_{2}+3 r u_{2}^{2}\right. \\
& \left.+3 r^{2} u_{2}-r u_{1}^{2}-u_{1}^{2} u_{2}-q^{2} u_{2}+u_{2}^{3}\right)-2 r \alpha \beta-\alpha \beta_{x}+\alpha_{x} \beta, \\
u_{2, t_{2}}= & -\frac{1}{2} \varepsilon q_{x x}-\varepsilon q^{3}+\varepsilon q r^{2}+(\varepsilon+1)\left(-\frac{1}{2} u_{1 x x}+2 q r u_{2}+2 r u_{1} u_{2}-3 q u_{1}^{3}\right. \\
& \left.+r^{2} u_{1}+q u_{2}^{2}+u_{1} u_{2}^{2}-3 q^{2} u_{1}-u_{1}^{3}\right)-2 q \alpha \beta+\alpha \alpha_{x}+\beta \beta_{x}, \\
\alpha_{t_{2}}= & \beta_{x x}-\frac{1}{2} q_{x} \alpha-\frac{1}{2} r_{x} \beta+\frac{1}{2} q^{2} \beta-\frac{1}{2} r^{2} \beta-q \alpha_{x}-r \beta_{x}, \\
\beta_{t_{2}}= & \alpha_{x x}+\frac{1}{2} r_{x} \alpha+\frac{1}{2} q_{x} \beta+\frac{1}{2} q^{2} \alpha-\frac{1}{2} r^{2} \alpha+r \alpha_{x}+q \beta_{x} .
\end{aligned}\right.
$$


Let $\varepsilon=0$ in (24), we have

$$
\left\{\begin{array}{l}
q_{t_{2}}=-\frac{1}{2} r_{x x}-q^{2} r+r^{3}+2 r \alpha \beta+\alpha \beta_{x}-\alpha_{x} \beta, \\
r_{t_{2}}=-\frac{1}{2} q_{x x}-q^{3}+q r^{2}+2 q \alpha \beta-\alpha \alpha_{x}-\beta \beta_{x}, \\
u_{1, t_{2}}=-\frac{1}{2} u_{2 x x}-2 q r u_{1}-2 q u_{1} u_{2}+3 r u_{2}^{2}+3 r^{2} u_{2}-r u_{1}^{2}-u_{1}^{2} u_{2}-q^{2} u_{2}+u_{2}^{3}-2 r \alpha \beta-\alpha \beta_{x}+\alpha_{x} \beta, \\
u_{2, t_{2}}=-\frac{1}{2} u_{1 x x}+2 q r u_{2}+2 r u_{1} u_{2}-3 q u_{1}^{3}+r^{2} u_{1}+q u_{2}^{2}+u_{1} u_{2}^{2}-3 q^{2} u_{1}-u_{1}^{3}-2 q \alpha \beta+\alpha \alpha_{x}+\beta \beta_{x}, \\
\alpha_{t_{2}}=\beta_{x x}-\frac{1}{2} q_{x} \alpha-\frac{1}{2} r_{x} \beta+\frac{1}{2} q^{2} \beta-\frac{1}{2} r^{2} \beta-q \alpha_{x}-r \beta_{x}, \\
\beta_{t_{2}}=\alpha_{x x}+\frac{1}{2} r_{x} \alpha+\frac{1}{2} q_{x} \beta+\frac{1}{2} q^{2} \alpha-\frac{1}{2} r^{2} \alpha+r \alpha_{x}+q \beta_{x} .
\end{array}\right.
$$

Especially, taking $\alpha=\beta=0$ in (24), we can obtain the nonlinear integrable couplings of the second order integrable equations

$$
\left\{\begin{array}{l}
q_{t_{2}}=-\frac{1}{2} r_{x x}-q^{2} r+r^{3} \\
r_{t_{2}}=-\frac{1}{2} q_{x x}-q^{3}+q r^{2} \\
u_{1, t_{2}}=-\frac{1}{2} \varepsilon r_{x x}-\varepsilon q^{2} r+\varepsilon r^{3}+(\varepsilon+1)\left(-\frac{1}{2} u_{2 x x}-2 q r u_{1}-2 q u_{1} u_{2}+3 r u_{2}^{2}+3 r^{2} u_{2}-r u_{1}^{2}-u_{1}^{2} u_{2}-q^{2} u_{2}+u_{2}^{3}\right) . \\
u_{2, t_{2}}=-\frac{1}{2} \varepsilon q_{x x}-\varepsilon q^{3}+\varepsilon q r^{2}+(\varepsilon+1)\left(-\frac{1}{2} u_{1 x x}+2 q r u_{2}+2 r u_{1} u_{2}-3 q u_{1}^{3}+r^{2} u_{1}+q u_{2}^{2}+u_{1} u_{2}^{2}-3 q^{2} u_{1}-u_{1}^{3}\right) .
\end{array}\right.
$$

If setting $\varepsilon=-1, u_{1}=-q, u_{2}=-r$ in (24), we obtain the second order super integrable equations of (17)

$$
\left\{\begin{array}{l}
q_{t_{2}}=-\frac{1}{2} r_{x x}-q^{2} r+r^{3}+2 r \alpha \beta+\alpha \beta_{x}-\alpha_{x} \beta \\
r_{t_{2}}=-\frac{1}{2} q_{x x}-q^{3}+q r^{2}+2 q \alpha \beta-\alpha \alpha_{x}-\beta \beta_{x}, \\
\alpha_{t_{2}}=\beta_{x x}-\frac{1}{2} q_{x} \alpha-\frac{1}{2} r_{x} \beta+\frac{1}{2} q^{2} \beta-\frac{1}{2} r^{2} \beta-q \alpha_{x}-r \beta_{x}, \\
\beta_{t_{2}}=\alpha_{x x}+\frac{1}{2} r_{x} \alpha+\frac{1}{2} q_{x} \beta+\frac{1}{2} q^{2} \alpha-\frac{1}{2} r^{2} \alpha+r \alpha_{x}+q \beta_{x} .
\end{array}\right.
$$

\section{Conclusion}

In this paper, we introduced an approach for constructing nonlinear integrable couplings of super integrable hierarchy. Zhang [17] once employed two kinds of explicit Lie algebra $F$ and $G$ to obtain the nonlinear integrable couplings of the GJ hierarchy and Yang hierarchy, respectively. It is easy to see that Lie algebra $F$ given in [17] is isomorphic to the Lie algebra span $\left\{e_{1}, e_{2}, e_{3}, e_{4}, e_{5}, e_{6}\right\}$ in $g l(6,2)$. So we can obtain nonlinear integrable couplings of super GJ and Yang hierarchy easily. The method in this paper can be applied to other super integrable systems for constructing their super integrable couplings.

\section{Acknowledgements}

This work was supported by the Natural Science Foundation of Henan Province (No.132300410202), the Science and Technology Key Research Foundation of the Education Department of Henan Province (No. 14A110010), the Youth Backbone Teacher Foundation of Shangqiu Normal University(No. 2013GGJS02). 


\section{References}

[1] Kupershmidt, B.A. (1985) Odd and Even Poisson Brackets in Dynamical Systems. Letters in Mathematical Physics, 9, 323-330. http://dx.doi.org/10.1007/BF00397758

[2] Li, Y.S. and Zhang, L.N. (1988) A Note on the Super AKNS Equations. Journal of Physics A: Mathematical and General, 21, 1549-1552. http://dx.doi.org/10.1088/0305-4470/21/7/017

[3] Tu, M.H. and Shaw, J.C. (1999) Hamiltonian Structures of Generalized Manin-Radul Super-KdV and Constrained Super KP Hierarchies. Journal of Mathematical Physics, 40, 3021-3034. http://dx.doi.org/10.1063/1.532741

[4] Liu, Q.P. and Hu, X.B. (2005) Bilinearization of N = 1 Supersymmetric Korteweg de Vries Equation Revisited. Journal of Physics A: Mathematical and General, 38, 6371-6378. http://dx.doi.org/10.1088/0305-4470/38/28/009

[5] Aratyn, H., Nissimov, E. and Pacheva, S. (1999) Supersymmetric Kadomtsev-Petviashvili Hierarchy: “Ghost” Symmetry Structure, Reductions, and Darboux-Bäcklund Solutions. Journal of Mathematical Physics, 40, 2922-2932. http://dx.doi.org/10.1063/1.532736

[6] Morosi, C. and Pizzocchero, L. (1993) On the Bi-Hamiltonian Structure of the Supersymmetric KdV Hierarchies. A Lie Superalgebraic Approach. Communications in Mathematical Physics, 158, 267-288. http://dx.doi.org/10.1007/BF02108075

[7] Hu, X.B. (1997) An Approach to Generate Superextensions of Integrable Systems. Journal of Physics A: Mathematical and General, 30, 619-632. http://dx.doi.org/10.1088/0305-4470/30/2/023

[8] He, J.S., Yu, J., Zhou, R.G. and Cheng, Y. (2008) Binary Nonlinearization of the Super AKNS System. Modern Physics Letters B, 22, 275-288. http://dx.doi.org/10.1142/S0217984908014778

[9] Liu, Q.P., Popowicz, Z. and Tian, K. (2010) Supersymmetric Reciprocal Transformation and Its Applications. Journal of Mathematical Physics, 51, Article ID: 093511. http://dx.doi.org/10.1063/1.3481568

[10] Ma, W.X., Xu, X.X. and Zhang, Y.F. (2006) Semi-Direct Sums of Lie Algebras and Continuous Integrable Couplings. Physics Letters A, 351, 125-130. http://dx.doi.org/10.1016/j.physleta.2005.09.087

[11] Guo, F.K. and Zhang, Y.F. (2005) The Quadratic-Form Identity for Constructing the Hamiltonian Structure of Integrable Systems. Journal of Physics A: Mathematical and General, 38, 8537-8548. http://dx.doi.org/10.1088/0305-4470/38/40/005

[12] Zhang, Y.F. and Tam, H.W. (2010) Four Lie Algebras Associated with $\mathrm{R}^{6}$ and Their Applications. Journal of Mathematical Physics, 51, Article ID: 093514. http://dx.doi.org/10.1063/1.3489126

[13] Ma, W.X. (2011) Nonlinear Continuous Integrable Hamiltonian Couplings. Applied Mathematics and Computation, 217, 7238-7244. http://dx.doi.org/10.1016/j.amc.2011.02.014

[14] You, F.C. (2011) Nonlinear Super Integrable Hamiltonian Couplings. Journal of Mathematical Physics, 52, Article ID: 123510. http://dx.doi.org/10.1063/1.3669484

[15] Zhang, Y.F. and Zhang, H.Q. (2002) A Family of Integrable Systems of Liouville and Lax Representation, Darboux Transformation for Its Constrained Flows. Applied Mathematics and Mechanics, 23, 23-30.

[16] Shi, H. and Tao, S.X. (2011) A Super-Integrable Hierarchy and Its Super-Hamiltonian Structure. International Journal of Nonlinear Science, 10, 12-16.

[17] Zhang, Y.F. (2011) Lie Algebras for Constructing Nonlinear Integrable Couplings. Communications in Theoretical Physics, 56, 805-812. http://dx.doi.org/10.1088/0253-6102/56/5/03 